- Risk of a needlestick injury is reduced if safety syringes are used.

- UK dental schools are changing over to safety syringes.

- All dental staff should be considering changing to safety syringes.

- Changing over does require investment of time in training.

\title{
Use of safety dental syringes in British and Irish dental schools
}

\author{
J. M. Zakrzewska ${ }^{1}$ and E. C. Boon ${ }^{2}$
}

\begin{abstract}
Aim The aim of the present study was to determine what types of dental syringes were being used in British dental schools and whether recent studies on the use of safety syringes had had any impact.

Introduction In 2001 a controlled trial showed that avoidable needlestick injuries could be reduced with the introduction of safety syringes which did not require the re-sheathing or removal of a needle from its syringe.

Method A self complete questionnaire asking about safety syringe use was distributed through the deans of all 16 dental schools in the UK and Ireland.

Results Fifteen schools formally replied and data are available for the missing one. Two schools have totally converted to the use of safety syringes and in seven schools some departments are using them. Six schools are not considering a change, four others are hoping to change and four are undecided as to whether they are going to change. Five schools had tried them previously. All acknowledge that extensive training is essential, there is also considerable staff resistance and the safety syringes currently available are still not ideal.

Conclusion All dental schools should determine their avoidable needlestick injuries rates, reconsider their views on the use of safety syringes and contribute to the development of the ideal model.
\end{abstract}

\section{BACKGROUND}

Dental healthcare workers are at high risk of sharps injuries and of these the most common are due to needlesticks. Needlestick injuries mean that staff may require prophylaxis and treatment, that if found to have an infection they will be precluded from exposure prone procedures (the whole of clinical dentistry) and compensation will only be paid if there is documented evidence that the infection has come from a patient and is due to inadequate equipment or training. It has been found that the commonest needlestick injury is that sustained during disposal of the needle

1*Senior Lecturer/Honorary Consulant in Oral Medicine, ${ }^{2}$ Dental Infection Control Nurse, Barts and the London, Queen Mary's School of Medicine and Dentistry

*Correspondence to: Dr Joanna M. Zakrzewska, Oral Medicine, Barts and the London,

Turner Street, London E1 2AD

Email J.M.Zakrzewska@qmul.ac.uk

\section{Refereed paper}

Received 07.08.02; Accepted 13.12.02

doi:10.1038/sj.bdj.4810445

๑ British Dental Journal 2003; 195: 207-209 and syringe. This has led to the US introducing legislation which makes it mandatory to use "devices specifically engineered to prevent injuries with contaminated sharp instruments.'

A device or system that would reduce the risk of needlestick injuries is therefore needed. It has led to the first major redesign of the dental syringe since it was introduced in 1921. The dental syringe has been incredibly successful and fulfilled most of the requirements for such an instrument. However its major flaw is that the needle has to be removed from the syringe prior to sterilisation hence putting the operator at increased risk of injury during the dismantling process. The rising awareness of transfer of CJD through instruments is also putting increased pressure on the introduction of fully disposable instruments. These issues have led to the development of dental safety syringes. There are now at least four different types of safety syringes on the world market. This prompted Cuny et al. ${ }^{2}$ to carry out a survey on the efficacy of four different types in one US dental school. They reported that none of them were effective and they showed no fall in needlestick injuries as a result of their introduction. Another dental school in the UK evaluated four different types of syringes according to self set criteria and decided that one type did fulfil enough requirements to warrant introduction to a dental school. The study went on to show that avoidable needlestick injuries could be reduced with the introduction of safety syringes which did not require the re-sheathing or removal of a needle from its syringe. ${ }^{3}$ Avoidable needlestick injuries were defined as those that would not occur if the syringe did not require dismantling or where the needle was automatically protected. This study was considered to provide enough evidence for dental schools to begin considering a change of practice.

The aim of the present study was to see what impact the UK study had on clinical practice in the dental schools and what some of the barriers were that were encountered when changing to new dental syringes.

\section{METHOD}

A short self complete questionnaire comprising nine questions on two sides of A4 was prepared to ascertain the types of syringes in use in all UK dental schools, in year 2002 and whether any changes were planned for the future. Schools were asked whether the article on safety syringes published in 2001 in the $B D J^{3}$ provided evidence for change and whether it influenced their change. 
Those schools who had changed syringes were asked whether training had been provided to students or staff and who had provided it and what the response was to the new syringes. Schools were also asked if they knew the number of avoidable needlestick injuries that had been sustained in the school in a year before use of safety syringes and one year after. The questionnaire, abstract of the safety syringe paper, a covering letter and a self-addressed envelope was sent to the deans of 15 British and Irish dental schools with the request to pass them on to a senior clinician in charge of infection control. After six weeks a repeat request was sent to the schools that had not replied.

The editor of Evidence Based Dentistry was asked to comment whether the safety syringe paper contained enough evidence for practice to be changed.

The school which produced the original paper reviewed its rate of injury and the use of the safety syringe in the unit previously acting as control.

\section{RESULTS}

Questionnaires were returned from all but one dental school (93\%) but the type of syringes being used in the non replying school is known. The 16th school is our own. Not all the schools answered all the questions.

Table 1 shows the results from all the schools including our own and includes comments made on the forms. Two schools had totally converted to the use of safety syringes and another six schools reported departments who were using them. Eight schools were not using safety syringes, four of which had tried them but gave up because of poor experience. Six schools were not considering a change, of which three had tried the new safety syringes previously. Four others were hoping to change, of which one is a school that has previously tried them. Four other schools were undecided as to whether they were going to change. The two schools that had changed over completely had had both in-house training and commercial support from a company. All schools reported problems with the new syringes and there was considerable staff resistance to change. Only two schools reported a reduction in avoid- able needlestick injuries since the use of safety syringes, one of these had only partially changed. One school had reduced its needlestick injury rate by ensuring that dental nurses did not handle any syringes. Ten schools provided no data on number of injuries. In our own school the unit changing over to the new syringes has not reported any further injuries and the rest of the school has had two reports on injury. Both injuries were in temporary dental nurses who had not been trained, one of them had been attempting to dismantle the syringe. They were also not aware of the school policy that syringes should only be disposed of by the staff member using the syringe.

The editor of the journal Evidence Based Dentistry is of the opinion that there is enough evidence in the article, especially if the results have been sustained, to change to safety syringes in dental schools, and this view is supported by eight other schools. Three schools did not consider that this paper provided enough evidence and two were unsure. Three schools considered that the article had influenced them and a further five had also been influenced by their own experience and the awareness of the need to change.

\section{DISCUSSION}

This survey has found that there is an increased awareness among British and Irish dental schools of the need to consider the change to safety syringes. Schools who have clinically trained oral microbiologists are more likely to consider the change important based on their own experience. The two companies selling safety syringes in the UK report increasing interest and sales, which suggests that they are being increasingly used.

It is clear that a changeover is difficult to establish and hinges on two main issues. One is the provision of education which not only needs to increase knowledge of the ability of safety syringes to reduce needlestick injuries and how to use them effectively but which also needs to lead to a change in behaviour and attitudes. The second issue is the lack of the ideal syringe that incorporates all the features of the old syringe together with the new safety features. Problems with the new safety syringes do not help to convince sceptical colleagues.

Table 1 Use of safety syringes in 14 British and 2 Irish dental schools - survey February 2001

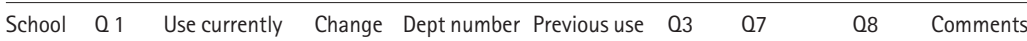

\begin{tabular}{|c|c|c|c|c|c|c|c|c|c|}
\hline 1 & 3 & Mixed & 2 & 1 & 1 & 0 & NA & NA & Tried them but not satisfied, have stopped nurses disposing of syringes. \\
\hline 2 & 1 & Old & 1 & 0 & 1 & 2 & NA & NA & About to change company will be helping to train staff. \\
\hline 3 & 3 & Old & 2 & 0 & 1 & 4 & NA & $\mathrm{Na}$ & Teach no touch technique. \\
\hline 5 & 1 & Old & 2 & 0 & 1 & 2 & 4 & 1 & Tried them and went back to old syringes. \\
\hline 6 & 0 & Old & 2 & 0 & 1 & 0 & NA & 0 & Tried but the new syringes were found to be too bulky. \\
\hline 7 & 2 & Mixed & 1 & 5 & 1 & 3 & 2 & 2 & Difficult to get new syringe accepted, staff resistance, but going to change. \\
\hline 8 & & Mixed & 1 & & & & & & No formal reply. \\
\hline 9 & 3 & Mixed & 3 & 3 & 3 & 3 & NA & NA & No single policy about use of syringes. \\
\hline 10 & 1 & Old & 2 & 0 & 1 & 4 & NA & NA & \\
\hline 11 & 1 & Mixed & 3 & 2 & 1 & 2 & 2 & 2 & Article did influence their decisions, all staff help to train others. \\
\hline 12 & 1 & New & 4 & 7 & 2 & 1 & 2 & 2 & Practise needed, variable quality of sheath, disengages, complaints about quality. \\
\hline 13 & 1 & New & 4 & 7 & 2 & 1 & 2 & 2 & All now changed, problems with temporary new staff as not always trained. \\
\hline 14 & 2 & Old & 2 & 0 & 1 & 4 & NA & NA & Change not been considered necessary and article would not convince. \\
\hline 16 & 2 & Old & 3 & 0 & 1 & 4 & NA & NA & Need training before introduction, tried safety syringes 1999 no change to injuries. \\
\hline
\end{tabular}

Question 1 Is there enough evidence in the article to consider a change? 1 - yes, 2 - no, 3 - unsure

Use currently old - non disposable syringes, mixed - safety syringe and non disposable, new - only safety syringe

Change 1 - consider changing, 2 - not considering change, 3 - do not know, 4 - changed

Dept using - number of departments using new syringe, maximum 7.

Previous use (prior to 2000) 1 - non disposable syringes, 2 - safety syringes, 3 - mixed

Question 3 What influenced change? 1 - own experience, 2 - reading article, 3 -other or mixture of 1 and 2

Question 7 Satisfaction with change to safety syringe? 1 - very, 2 - moderately, 3 - indifferent, 4 - not satisfied

Question 8 Difficulties encountered during change? 1 - great, 2 - some, 3 - none

NA - not applicable 
Training, preferably with the help of the manufacturers, appears to improve introduction of the new system throughout the whole school. Continual training as new staff, both permanent and temporary, join the schools is imperative until the new safety syringes become the norm. Dental nurses continue to sustain injuries from syringes as shown in our dental school and also in a recent survey among British dental nurses which showed that over $60 \%$ of nurses responding to a questionnaire reported sustaining at least two injuries during their employment. ${ }^{4}$ This has prompted one school to ensure that syringes are not handled by nurses and has led to a reduction in injuries. Change has to begin somewhere and dental schools are well suited to introducing such changes as they are large and can monitor outcome. ${ }^{2}$ All dental students in our school are taught the use of the new syringes and all are assessed on their ability to use them during a practical which counts towards their internal assessment. We have ensured that students not reaching the standard are re-trained and we have not had a single report of a dental student injury using the new safety syringe. Another measure of success of the new safety syringes is to assess how the number and pattern of needlestick injuries has changed from baseline. It was disappointing to see the lack of details on numbers of needlestick injuries sustained in the dental schools as it should be considered part of the role of risk management departments to assess injuries and determine what measures can be taken to reduce them.

Cuny et $a .^{2}$ have shown that there is dissatisfaction with the design of new safety syringes and this is also supported by this survey, with five schools reporting going back to the old syringes due to problems with the new syringes. The manufacturers for the UK market have been aware of this and continue to introduce substantial changes to their designs to meet consumers' criticisms. Both models have undergone changes since last reported on in the study by Zakrzewska et al. $^{3}$ It is essential that consumers do report back their concerns and it may be time to perform a survey of consumers views on the new safety dental syringes using the criteria set out by Cuny et al. ${ }^{2}$ This, in the long run, will mean an improved product and improved safety for all staff.

The increased pressure to use fully disposable instruments due to the risks of transmission of CJD and the need to protect staff from avoidable injuries, mean that the disposable safety syringe is here to stay. All dental schools should determine their avoidable needlestick injuries rates, reconsider their views on the use of safety syringes and contribute to the development of a dental syringe that can be used for the next few decades.

The authors are very grateful to all the dental schools for answering the questionnaire, as we know just how many requests they receive. We are grateful to both Kavo and Septodont for their continued support in our quest to find the ideal safe dental syringe.

E.C. Boon is sponsored by Septodont

1. Voelker R. Needlestick Bill Passes. J Am Med Assoc 2000; 284: 2585.

2. Cuny $E_{1}$ Fredekind R E, Budenz A W. Dental safety needles' effectiveness: results of a one-year evaluation. J Am Dent Assoc 2000; 131: 1443-1448.

3. Zakrzewska J M, Greenwood I, Jackson J. Introducing safety syringes into a UK dental school-a controlled study. Br Dent J 2001; 190: 88-92.

4. Ower P, Jones S. Needlestick injuries- is there an answer? Independent Dent 2000; 3 : $78-80$ 